\title{
Advanced Measuring (Instrumentation) Methods for Nuclear Installations
}

\author{
Yan Yang, ${ }^{1}$ Jin Huang, ${ }^{1}$ and Xing Chen ${ }^{2}$ \\ ${ }^{1}$ College of Mechanical Engineering, Chongqing University of Technology, Chongqing 400054, China \\ ${ }^{2}$ Department of Mechanical Engineering, Chonnam Naitional University, Gwangju 500-757, Republic of Korea
}

Correspondence should be addressed to Yan Yang, yangyan@cqut.edu.cn

Received 22 November 2012; Accepted 22 November 2012

Copyright (C) 2012 Yan Yang et al. This is an open access article distributed under the Creative Commons Attribution License, which permits unrestricted use, distribution, and reproduction in any medium, provided the original work is properly cited.

In last decades, nuclear technology has developed rapidly and become more important to human society. Nuclear technology has many advantages, such as zero carbon emissions, energy independence, and safety. At present, nuclear installations are more prevalent than ever before. Therefore, with the rapid development and wide applications of nuclear technology, many new technologies have been emerging to guarantee its reliability and safety, where measuring devices and techniques that can exactly measure and monitor the nuclear installations show particular importance.

The research of measurement in nuclear installations involves many aspects, such as nuclear reactors, nuclear fuel cycle, safety and security, and environmental applications. Recently, a big number of advanced measuring devices and techniques have been widely applied in nuclear installations. Multifarious nuclear radiation detectors were used in nuclear radiation detection. A variety of measuring instruments, for example, mechanical gauges, flow meters, pressure and temperature sensors, were employed to control the systems, monitor possible development of defects/failures, and especially to prevent catastrophic accidents.

In this special issue, we published 9 articles. All contributions highlight recent major breakthroughs, progress, and challenges on instrumentation and measuring methods in nuclear installations. In these articles, one is the review paper of advance measuring methods and instrumentations for nuclear installations, four papers are concerned with measurement method and four papers present some new measurement instrumentations.

The review paper "Advanced measuring (instrumentation) methods for nuclear installations: a review" mainly introduces the development of the measuring (instrumentation) methods for nuclear installations and the applications of these instruments and methods.

For spacer grid in PWR fuel, previous research on the strength analyses was performed using base material properties owing to a lack of mechanical properties in the weld zone. The paper "Effect of weld properties on the crush strength of the PWR spacer grid" analyzed the strength considering the mechanical properties in the weld zone and obtained satisfied results.

The paper "Nuclear power plant instrumentation fault detection using fuzzy logic" discussed the availability of a large number of measured signals and additional component information, and the increasing number of signal processing options to analyze sampled data motivates the assimilation of such diverse information into a plantwide condition monitor. The use of fuzzy logic is described for the purpose of performing the decision making regarding the system status and the possible need for component maintenance. Fuzzy-logic-based diagnostic monitoring is applied to data acquired from instrumentation within operating facilities.

A new estimation method to determine the optimal number of transducers for accurate flow rate measurement downstream of a single elbow is presented by "Study on the optimal number of transducers for pipe flow rate measurement downstream of a single elbow using the ultrasonic velocity profile method." The new estimation method employs a wave number of velocity profile fluctuations along a circle on a pipe cross-section using fast fourier transform. The optimal number of transducers is estimated based on the sampling theorem. To evaluate this method, a preliminary 
experiment and numerical simulations using computational fluid dynamics are conducted. The results indicate the efficiency of this new method.

Another new method of NCT for a sample liver phantom was defined by the paper "Design and simulation of a new model for treatment by NCT." An interaction of $12 \mathrm{MeV}$ neutrons with a multilayer spherical phantom is considered. The phantom consists of equivalent liver tissue at the centre, covered with a layer of polyethylene and an external thin layer of cadmium. In order to reach the desirable energy range of neutrons in accord with required energy in absence of eligible clinical neutron source for NCT, this model of phantom might be utilized. The neutron flux and the deposited dose in all components and different layers of the mentioned phantom are computed by Monte Carlo simulation.

Besides many new measurement methods, more novel instruments were widely used for measurement in nuclear techniques. In order to diagnose laser-plasma distribution in space and evolution in time, an elliptical curved crystal spectrometer has been developed by the paper " $A$ novel spectrometer for measuring laser-produced plasma X-ray in inertial confinement fusion." The curved crystal spectrometer mainly consists of elliptical curved crystal analyzer, vacuum configuration, aligning device, spectral detectors, and three dimensional microadjustment devices. The spectrographic experiment was carried out on the XG-2 laser facility. Emission spectrums of Al plasmas, Ti plasma, and Au plasmas have been successfully recorded by using X-ray CCD camera.

A variety of new equipments based on smart materials were also used in measurement and controlling of nuclear installations. The paper "Squeezing force of the magnetorheological fluid isolating damper for centrifugal fan in nuclear power plant" reports the analytical endeavor into the fluid dynamic modeling of an MR isolating damper. The velocity and pressure distribution of the magnetorheological fluid operating in an axisymmetric squeeze model are analytically solved using a biviscosity constitutive model. Analytical solutions for the flow behavior of MR fluid flowing through the parallel channel are obtained. The equation for the squeezing force is derived to provide the theoretical foundation for the design of the isolating damper.

Centrifugal fan is an important component of ventilation system in nuclear power plant. A continuously variable transmission system based on magnetorheological fluid and shapememory alloy for improving the operating efficiency of the centrifugal fan in nuclear power plant was proposed by the paper "MR continuously variable transmission driven by SMA for centrifugal fan in nuclear power plant." The equation of transmission torque developed by MR fluids is derived to compute the torque transmission ability in the continuously variable transmission system. A shape-memory alloy spring actuator is designed to control the electric current in coil assembly. The results indicate that the change of temperatures has a tremendous influence on the electric current in coil assembly, the transmission torque of the continuously variable transmission system changes rapidly according to the temperatures acting on shape-memory alloy spring actuator, and the output angular velocity of the centrifugal fan can be adjusted continuously.
A theoretical analysis of the effect of the applied magnetic field on the viscoplastic flow between two cylinders in the speed-adjustment system is presented by "Cylindrical magnetorheological fluid variable transmission controlled by shapememory alloy." A sliding mode SMA switch is proposed to modify the magnetic field acting on working gap under thermal effect.

In this special issue, the development of the measuring (instrumentation) methods for nuclear installations and the applications of these instruments and methods are presented. Various instruments and measurement methods used in nuclear power reactors, nuclear fuel cycle, safeguards, and analysis are discussed in detail. With the rapid development and wide applications of nuclear technology, more and more novel technologies will be used for measurement and monitoring in nuclear installations.

Yan Yang Jin Huang Xing Chen 

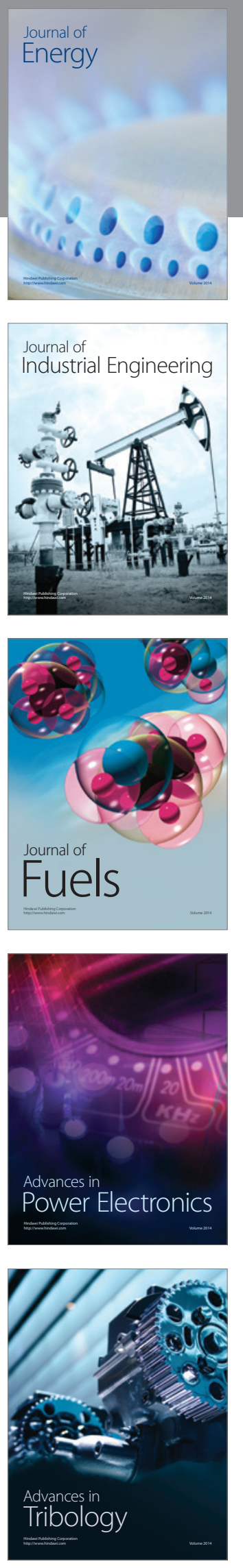
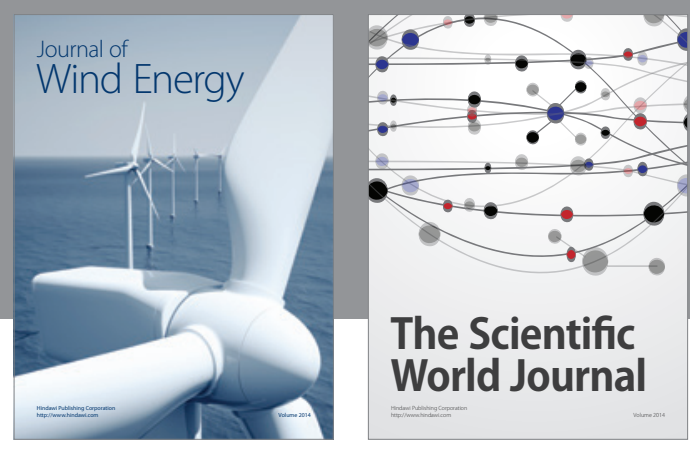

The Scientific World Journal

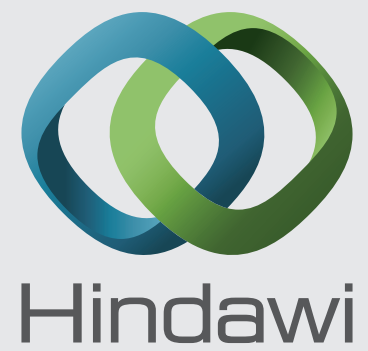

Submit your manuscripts at http://www.hindawi.com
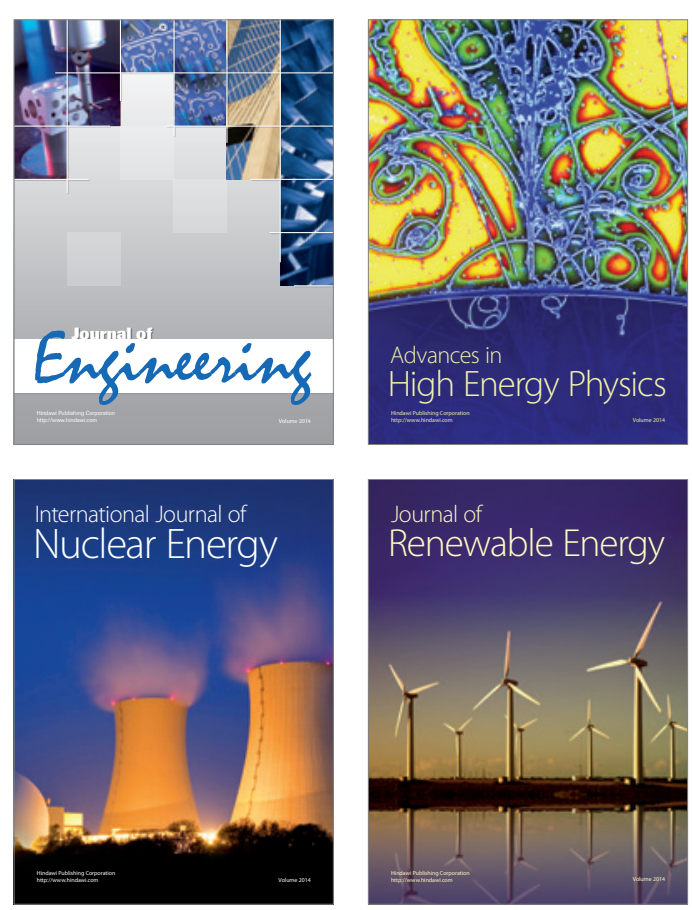

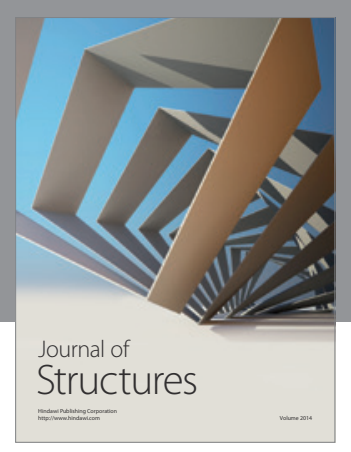

Rotating
Mechinery
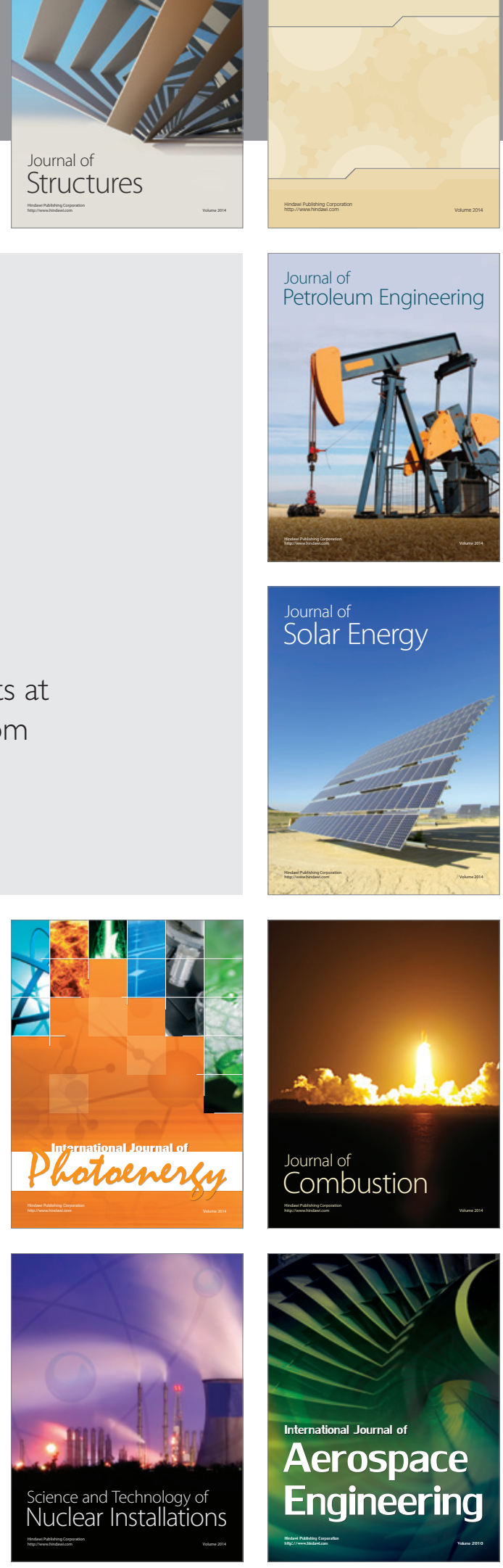